\title{
Mechanism of TSCS promote anti-Fas antibody-induced FLS apoptosis via Blocking MC-Tryptase-PAR-2-Rho-FLS Signaling Pathway
}

\section{Shi-Gang Li}

Medical School, China Three Gorges University, China

It has been supposed that mast cells have important participation in the physiopathology of RA,however, the role of mast cells in the pathogenesis of RA remains unclear.

In this study, we observed the antiapoptotic effects of tryptase released by mast cell on RA synovial fibroblasts, and found that tryptase significantly increased the expression of PAR2 on the surface of fibroblast like synovial cell,significantly activated Rho kinase and inhibited apoptosis of fibroblast like synovial cell induced by CH11.In the coculture system of mast cells and fibroblast like synovial cells, TSCS treatment significantly inhibited Rho kinase, significantly promoted apoptosis of fibroblast like synovial cell induced by $\mathrm{CH} 11$, These results demonstrate that tryptase may play a key role in the physiopathology of RA.TSCS can inhibit Mast cells activation and promote FLS cells apoptosis, which provide theoretical and experimental basis for the study of mast cells as targets for new antiRA drugs. 